\title{
Experimental and Spectral Finite Element Study of Plates with Shunted Piezoelectric Patches
}

\author{
M. Tawfik \\ Mathematics Department, German University in Cairo, New Cairo City, Cairo, Egypt
}

A. Baz

Mechanical Engineering Department, University of Maryland, College Park, MD, 20742, USA

(Received 10 March 2003; accepted 23 February 2004)

The need for passive damping techniques arises from the complexities, added weight, and energy requirements associated with the implementation of various active control techniques. A novel passive damping approach for the attenuation and localisation of the vibration of plates is presented in this study. The introduction of distributed piezoelectric patches with passively shunted circuits is presented. A numerical model that describes the coupling of a shunted circuit with flexible plates is developed using a spectral finite element approach. The prediction of the model is validated against analytical and experimental results. The numerical and experimental results obtained demonstrate the feasibility of using piezoelectric patches with passive shunting as an effective means for damping out the plate vibration. Furthermore, it is shown that the proposed approach is capable of producing broadband attenuation and localisation of the vibration by introducing disorder into the passive shunting circuits.

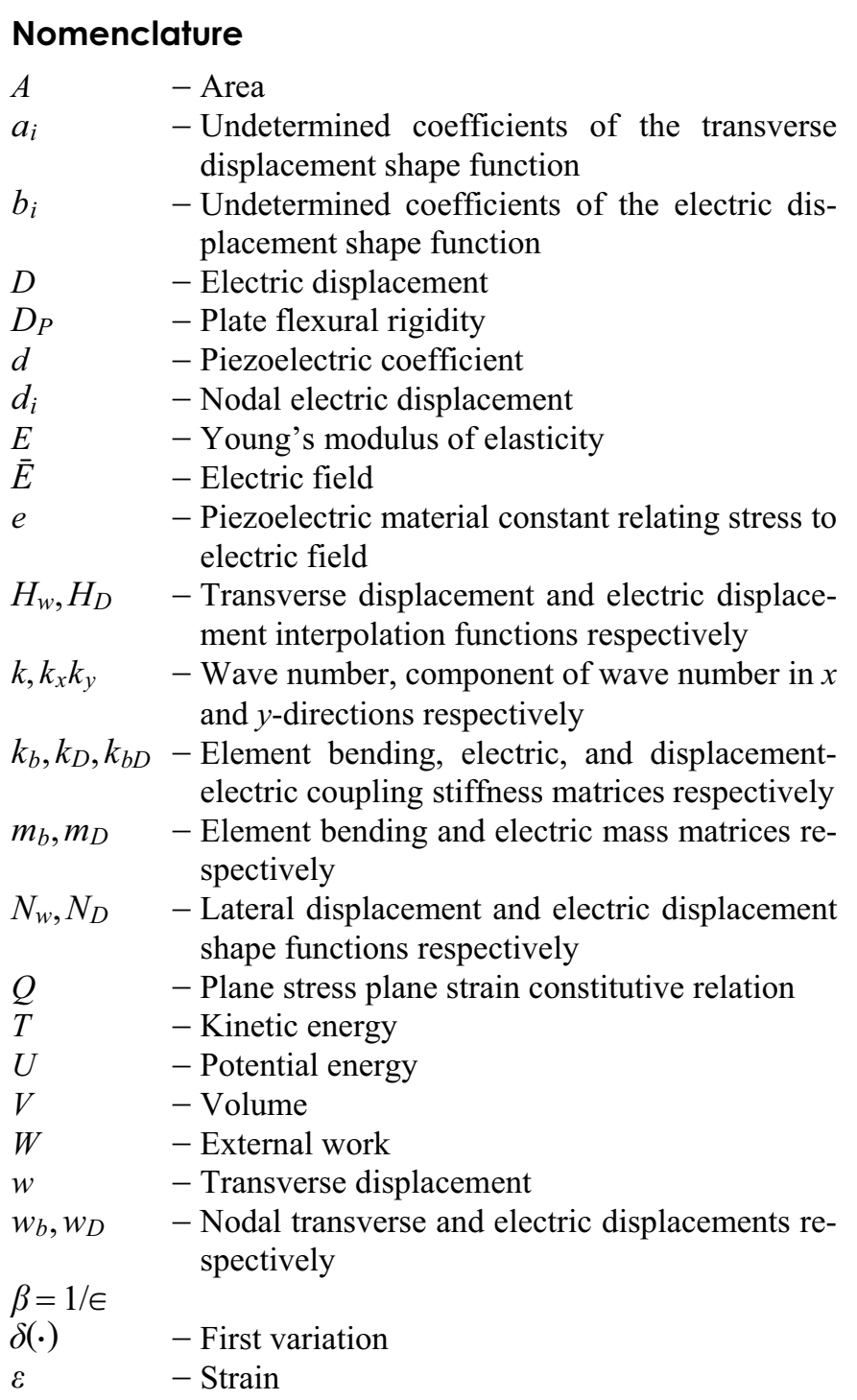

$\begin{array}{ll}\gamma_{x y} & - \text { Shear strain } \\ \kappa & - \text { Curvature } \\ \mu & - \text { The propagation constant } \\ \rho & - \text { Mass density } \\ \sigma & - \text { Stress } \\ \theta & - \text { Wave propagation angle } \\ v & - \text { Poisson's ratio } \\ \omega & - \text { Frequency } \\ \epsilon & - \text { Dielectric constant } \\ \vartheta & - \text { Phase angle }\end{array}$

\section{Subscripts}

$D$ - Related to electric degrees of freedom

$w$ - Related to transverse deflection

$b-$ Related to bending degrees of freedom

$x \quad$ - In the $x$-direction

,$x$ - Derivative in the $x$-direction

$y-$ In the $y$-direction

,$y$ - Derivative in the $y$-direction

\section{Superscript \\ $D$ - At constant electric displacement \\ $\bar{E}$ - At constant electric field \\ $T$ - Matrix transpose}

\section{INTRODUCTION}

\subsection{Passively Shunted Piezoelectrics}

In a review article about shunted piezoelectric elements, Lesieutre $^{1}$ presented a classification of shunted circuits into inductive, resistive, capacitive, and switched circuits. He emphasised that the inductive circuits, which include an inductor and a resistance in parallel with the piezo-capacitor (Fig. 1), are the most widely used circuits in damping as they are analogous to a mechanical vibration absorber. 